\title{
Capsule retention caused by duodenal metastases from primary appendiceal adenocarcinoma
}

\author{
Jen-Wei Chou ${ }^{1,2,3,4}$, Ken-Sheng Cheng ${ }^{1,2}$ \\ ${ }^{1}$ School of Medicine, China Medical University, Taichung, ${ }^{2}$ Division of Gastroenterology and Hepatology, Department of Internal Medicine, \\ China Medical University Hospital, Taichung, Taiwan, R.O.C; ${ }^{3}$ Taiwan Society of Inflammatory Bowel Disease; ${ }^{4}$ Taiwan Association for the \\ Study of Small Intestinal Diseases
}

\begin{abstract}
Metastatic small bowel cancers are extremely rare in clinical practice. Primary appendiceal adenocarcinoma with gastrointestinal metastasis is seldom reported in the literature. Here, we describe the case of an 80-year-old male patient with primary appendiceal adenocarcinoma, who presented to China Medical University Hospital with mid-gastrointestinal tract bleeding. Capsule endoscopy revealed stenotic bowel lumen, but the capsule was retained in the distal duodenum. Double-balloon enteroscopy demonstrated erosive and erythematous mucosa in the region of capsule retention. The retained capsule was retrieved successfully by using an electrosurgical snare. Histological examination and immunohistochemical staining of the biopsy specimen from the duodenal lesion strongly supported the diagnosis of metastatic appendiceal adenocarcinoma. (Intest Res 2017;15:130-132)
\end{abstract}

Key Words: Primary appendiceal adenocarcinoma; Metastatic small bowel cancer; Capsule endoscopy; Capsule retention; Double-balloon enteroscopy

\section{INTRODUCTION}

Small bowel tumors are rare causes of mid-gastrointestinal (GI) bleeding, accounting for $4 \%$ to $5 \%$ of all cases of GI bleeding. Metastatic small bowel cancers are extremely rare in clinical practice. Capsule endoscopy (CE) and doubleballoon enteroscopy (DBE) are useful modalities in the diagnosis of small bowel tumors. However, the occurrence rate of capsule retention is higher in small bowel tumors than in other small bowel conditions. Herein, we report the case of a primary appendiceal adenocarcinoma with duodenal metastases that presented with mid-GI bleeding. CE was performed for further investigation of obscure GI

Received July 23, 2016. Revised August 6, 2016.

Accepted August 22, 2016.

Correspondence to Ken-Sheng Cheng, Division of Gastroenterology and

Hepatology, Department of Internal Medicine, China Medical University

Hospital, No. 2, Yude Road, North District, Taichung 40447, Taiwan,

R.O.C. Tel: +886-4-22052121 (ext. 2220), Fax: +886-4-22023119, E-mail:

codecol80@gmail.com

Financial support: None. Conflict of interest: None. bleeding; however, capsule retention occurred in the distal duodenum. We performed antegrade DBE and retrieved the capsule by using an electrosurgical snare. Erosive and erythematous mucosa and a stenotic lumen were identified in the distal duodenum. Duodenal metastases from a known appendiceal adenocarcinoma were ultimately confirmed by pathological examination.

\section{CASE REPORT}

An 80-year-old man presented to China Medical University Hospital with a 1-week history of dark red stool. Moreover, he complained of poor appetite and weight loss of $14 \mathrm{~kg}$ for 2 months prior to admission. One year prior to this presentation, he experienced right lower abdominal pain and underwent an emergency appendectomy at a local hospital. The pathological examination revealed primary appendiceal adenocarcinoma, and he subsequently underwent a right hemicolectomy. Regional metastatic lymph nodes were identified at that time. He received oral chemotherapy at our outpatient department. On admission, physical examina-

\footnotetext{
๑ Copyright 2017. Korean Association for the Study of Intestinal Diseases. All rights reserved.

This is an Open Access article distributed under the terms of the Creative Commons Attribution Non-Commercial License (http://creativecommons.org/licenses/by-nc/4.0)

which permits unrestricted non-commercial use, distribution, and reproduction in any medium, provided the original work is properly cited.
} 
tion showed a man with thin stature. His conjunctivae were pale and his sclerae were anicteric. His abdomen was soft and scaphoid. The results of laboratory tests showed a hemoglobin level of $7.9 \mathrm{~g} / \mathrm{dL}$ (normal range, $14-16 \mathrm{~g} / \mathrm{dL}$ ) and a serum CEA level of $27.3 \mathrm{ng} / \mathrm{mL}$ (normal range, $<5 \mathrm{ng} / \mathrm{mL}$ ). Abdominal CT demonstrated multiple preaortic metastatic lymph nodes (Fig. 1, arrows). Esophagogastroduodenoscopy and colonoscopy found no bleeding source. Mid-GI bleeding was suspected, and wireless CE (PillCam SB; Given Imaging, Yokneam, Israel) was performed. However, capsule retention in the right upper quadrant was demonstrated by follow-up abdominal radiography. Antegrade DBE (EN-

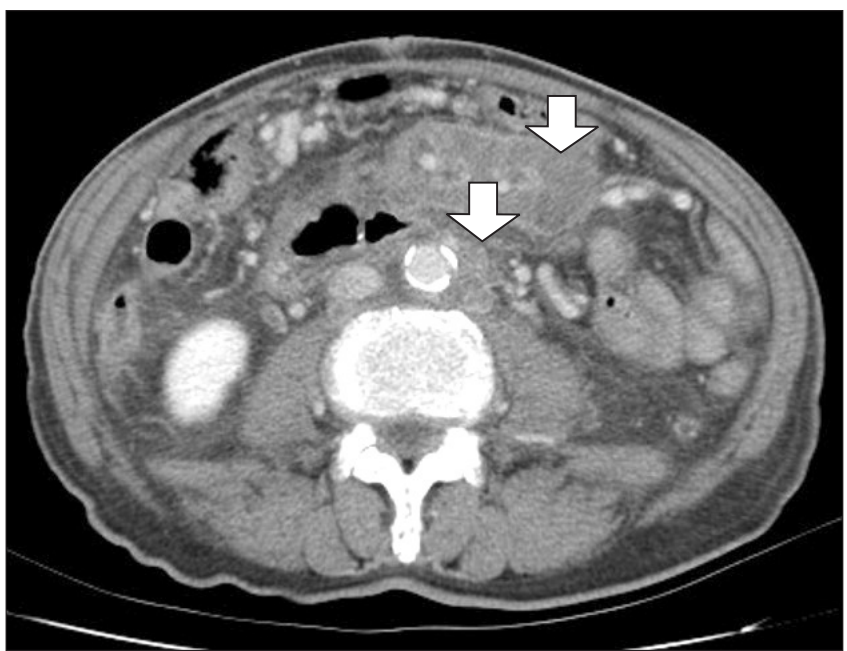

Fig. 1. Abdominal CT finding. CT revealed multiple enlarged preaortic lymph nodes (arrows).

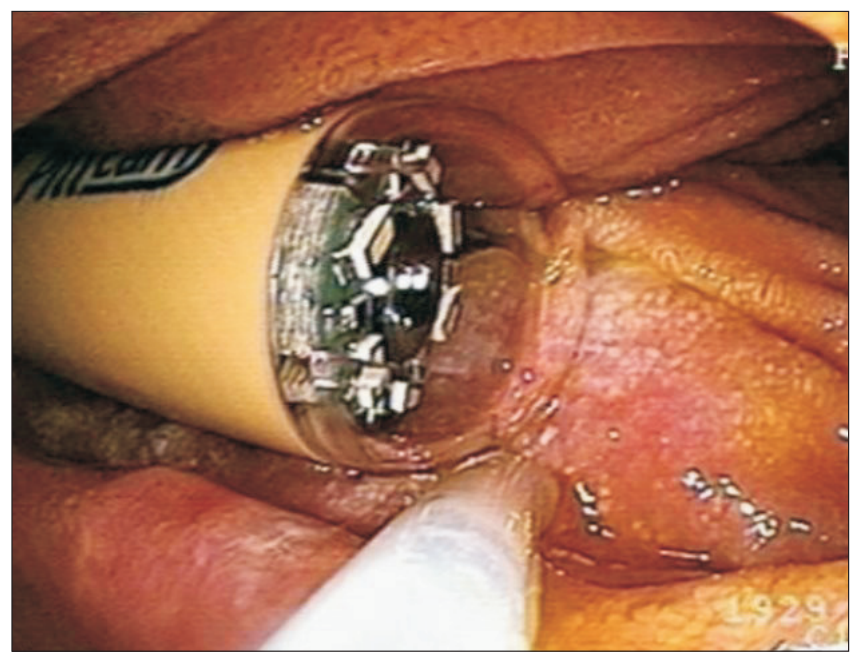

Fig. 2. Enteroscopic finding. Antegrade double-balloon enteroscopy demonstrating a retained capsule in the stenotic duodenal lumen.
450T5/20; Fujinon, Saitama, Japan) demonstrated a retained capsule in the third portion of the duodenum (Fig. 2). We used an electrosurgical snare to successfully retrieve the retained capsule. Erosive and erythematous mucosa and a stenotic lumen were identified in the distal duodenum (Fig 3). Multiple forceps biopsies were taken from the duodenal mucosal lesions. Histopathological examination of the biopsy specimens was consistent with poorly differentiated adenocarcinoma (Fig. 4). Immunohistochemical staining of the tumor cells was positive for CK20 but negative for CK7. On the basis of the pathological features, a diagnosis of duodenal metastases from primary appendiceal adenocar-

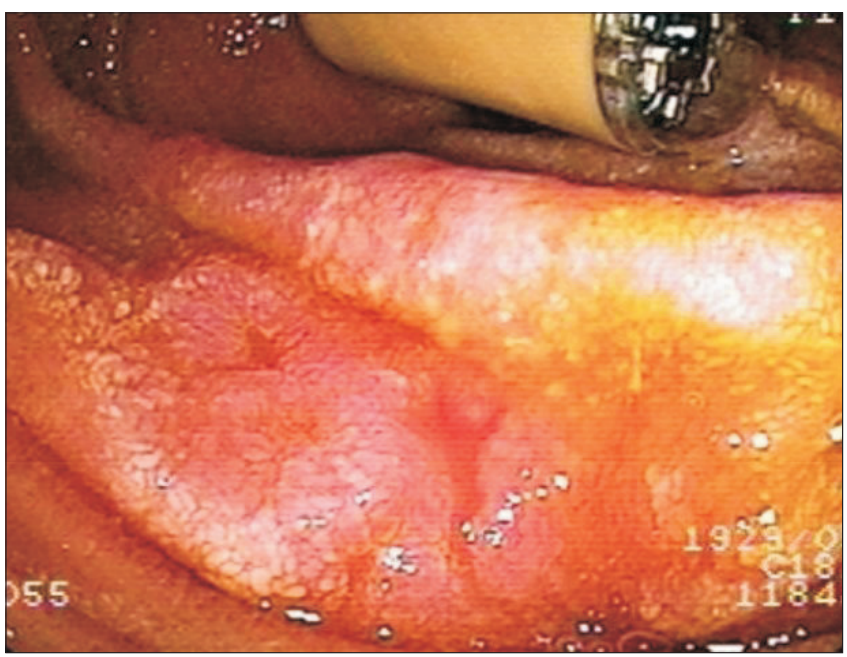

Fig. 3. Enteroscopic finding. Erosive and erythematous mucosa were identified in the stenotic lumen of the duodenum during doubleballoon enteroscopy.

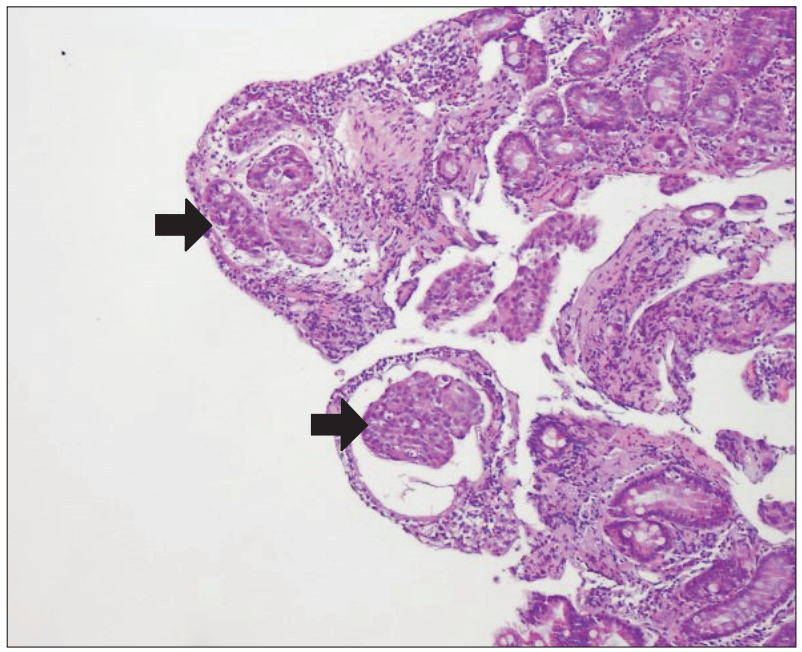

Fig. 4. Pathologic finding. Histopathological section of a biopsy specimen showing poorly differentiated metastatic adenocarcinoma (arrows) $(H E E E, \times 100)$. 
cinoma was made. The patient received palliative treatment because of his poor performance status. He died of multiple organ failure 1 month later.

\section{DISCUSSION}

Small bowel metastases usually originate from primary carcinomas of the GI system or genital organs, and more specifically from the large bowel, uterus, cervix, ovaries, and testes. ${ }^{1}$ Among metastatic small bowel cancers, melanomas, lung cancers, and breast cancers are the most common primary tumors. Metastatic small bowel cancers are usually asymptomatic; if symptomatic, they may present as a perforation, obstruction, intussusception, malabsorption, or hemorrhage. Although the appendix is a portion of the colon, primary appendiceal cancer is a rare event and is usually discovered after pathological examination of a surgically removed inflamed appendix. ${ }^{2}$

The age-adjusted incidence of primary appendiceal cancer is 0.12 cases per $1,000,000$ population per year. The incidence of metastatic colorectal cancer to the small intestine is about $6 \% .{ }^{3}$ However, metastatic primary appendiceal cancer to the GI tract is rare, and only two cases of gastric metastases have been reported. ${ }^{4,5}$ However, small bowel metastases caused by primary appendiceal cancer have never been reported in the literature. Our patient had histologically proven small intestinal lesions originating from primary appendiceal adenocarcinoma.

This is the first reported case of duodenal metastasisinduced GI bleeding from primary appendiceal adenocarcinoma. Metastatic cancers may involve the small intestine via contiguous spread, peritoneal metastases, or hematogenous metastases. In our patient, we hypothesized the development of duodenal metastases from primary appendiceal adenocarcinoma via direct invasion from metastatic preaortic lymph nodes, complicated by luminal stenosis, hemorrhage, and capsule retention. In the past, the diagnosis of small bowel tumors has been difficult and was often delayed for months after onset of symptoms because of delayed presentation, nonspecific signs and symptoms, and lack of accurate diagnostic studies.

In recent years, the development of enteroscopy and other diagnostic tools has enabled earlier nonoperative diagnosis. CE and DBE have been useful in the detection of small bowel lesions. ${ }^{6,7}$ However, capsule retention is a major complication of CE. The capsule retention rate in patients with obscure GI bleeding is $1.5 \%$, but is higher $(5 \%)$ in patients with known CD. ${ }^{8}$ However, capsule retention occurs in $9.8 \%$ of patients with small bowel tumors. ${ }^{9}$ In contrast to CE, DBE not only provides good diagnostic yield for small bowel lesions, but also enables tissue sampling and treatment. Moreover, DBE is a reliable method for removing retained capsules and might prevent unnecessary surgery. ${ }^{10}$ The treatment of metastatic small bowel cancers usually depends on the nature of the primary tumor. The treatment options for metastatic disease include systemic chemotherapy alone, hyperthermic intraoperative intraperitoneal chemotherapy, cytoreductive surgery with peritonectomy, and a combination of treatments. Of those treatment options, cytoreductive surgery and hyperthermic intraperitoneal chemotherapy have recently become the treatment of choice for metastatic diseases at most large centers. Even though radical resection of small bowel cancer plays an important role, the 5-year overall survival rate remains low.

In conclusion, although mid-GI bleeding induced by metastatic small bowel tumors is rare, it should be considered in patients with a known malignancy.

\section{REFERENCES}

1. Gill SS, Heuman DM, Mihas AA. Small intestinal neoplasms. J Clin Gastroenterol 2001;33:267-282.

2. Ko YH, Jung CK, Oh SN, et al. Primary signet ring cell carcinoma of the appendix: a rare case report and our 18-year experience. World J Gastroenterol 2008;14:5763-5768.

3. Thoma MN, Saiyed SM, Charles RJ. Metastatic colorectal cancer to the small bowel-an uncommon cause of obscure GI bleeding: a three-case experience with review of the literature. J Gastrointest Cancer 2011;42:119-122.

4. Miyashita T, Hoshino E, Imamura T, Okinaga K, Akaoka I, Miyashita $\mathrm{H}$. A case of appendiceal cancer metastatic to the stomach with pseudomyxoma peritonei. J Gastroenterol 1994;29:71-75.

5. Yoshida R, Yoshioka K, Yoshitaka H, et al. Pseudomyxoma peritonei of appendiceal cancer with metastasis to the stomach: report of a case. Surg Today 2002;32:547-550.

6. Schwartz GD, Barkin JS. Small-bowel tumors detected by wireless capsule endoscopy. Dig Dis Sci 2007;52:1026-1030.

7. Yamamoto H, Sekine Y, Sato Y, et al. Total enteroscopy with a nonsurgical steerable double-balloon method. Gastrointest Endosc 2001;53:216-220.

8. Lewis B. How to prevent endoscopic capsule retention. Endoscopy 2005;37:852-856.

9. Rondonotti E, Pennazio M, Toth E, et al. Small-bowel neoplasms in patients undergoing video capsule endoscopy: a multicenter European study. Endoscopy 2008;40:488-495.

10. Van Weyenberg SJ, Van Turenhout ST, Bouma G, et al. Doubleballoon endoscopy as the primary method for small-bowel video capsule endoscope retrieval. Gastrointest Endosc 2010;71:535-541. 